\title{
2013 Morgan Prize
}

FAN WEI received the 2013 AMS-MAA-SIAM Frank and Brennie Morgan Prize for Outstanding Research in Mathematics by an Undergraduate Student at the 119th Annual Meeting of the AMS in San Diego, California, in January 2013. Receiving honorable mentions were DHRUV RANGANATHAN and JONATHAN SCHNEIDER.

\section{Citation}

Fan Wei is awarded the 2013 AMS-MAA-SIAM Frank and Brennie Morgan Prize for Outstand-

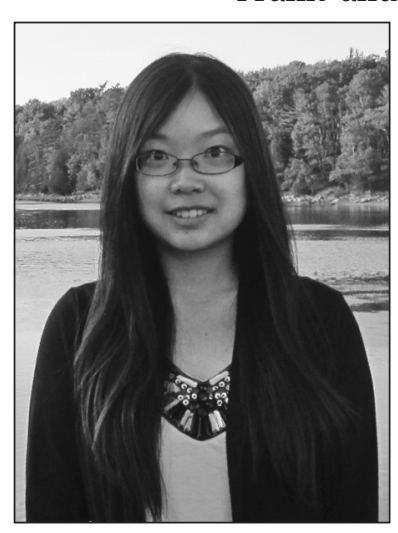
ing Research in Mathematics by an Undergraduate Student for her wide range of scholarly contributions. As an undergraduate at the Massachusetts Institute of Technology, Wei authored or coauthored five papers in fields as diverse as number theory, combinatorics, statistics, and tropical geometry, and she is recognized, in particular, for her single-authored paper that solves a separable permutations problem posed by Dr. Richard Stanley. Her work has been described as "impressive and ingenious" and as

Fan Wei "enthusiastically received by other mathematicians."

Wei has attended REUs at Williams College and the University of Minnesota-Twin Cities and has participated in many research projects at MIT. She has presented her results at two conferences in 2010: the Young Mathematician's Conference and Permutation Patterns.

Wei was part of a Meritorious Winner Team for the 2010 Mathematical Contest in Modeling, a mentor of the Girl's Angle Math Club in Cambridge, and has served on the board of MIT's Society of Women Engineers. Additionally, Wei won the 2012 Alice T. Schafer Prize of the Association for Women in Mathematics (AWM).

\section{Biographical Sketch}

Fan Wei is from Beijing, China, where she finished high school and became interested in mathematics. In 2012, Fan Wei received her bachelor's degree from the Massachusetts Institute of Technology, where she majored in mathematics and became more committed to the subject. Her interests in mathematics are in analysis and combinatorics. After a summer

DOI: http://dx.doi.org/10.1090/noti968 internship at Microsoft Research New England with Henry Cohn, she went to Cambridge University, where she is studying Part III mathematics for a master's degree. She plans to return to the United States to obtain a Ph.D.

\section{Response}

I am very honored and grateful to receive the Frank and Brennie Morgan Prize. It is a great encouragement for me and I would like to thank the AMS, MAA, and SIAM for selecting me for this award.

First and foremost, I want to thank my parents for their constant love, understanding, and tolerance. My home has always been, and will continue to be, my motivation. My gratitude goes to my research mentors, class lecturers, and nominators, Richard Dudley and Richard Stanley. Furthermore, I want to express my gratitude to Henry Cohn, my mentor at Microsoft Research; to the hosts of the UMN REU—Gregg Musiker, Victor Reiner, and Pavlo Pylyavskyy; and to the hosts of Williams College SMALL REU, especially Allison Pacelli, for providing me with two memorable summers. I am also grateful to the MIT mathematics department and the many people, including the staff members and professors, such as Michael Artin, Alan Edelman, Ju-Lee Kim, Gigliola Staffilani, Daniel Stroock, and other analysis and combinatorics professors, for their great help, patience, and support. Lastly, I want to thank all my friends for giving me a second family. I am lucky to know you all.

\section{Citation for Honorable Mention: Dhruv Ranganathan}

Honorable mention goes to Dhruv Ranganathan for the 2013 AMS-MAA-SIAM Frank and Brennie Morgan Prize for Outstanding Research in Mathematics by an Undergraduate Student. Ranganathan is recognized for his research in Gromov-Witten theory and toric geometry and, in particular, for his article "Toric symmetry of $C P^{3}$ ". Letter writers remarked on his ability to "master the formidable prerequisite material" and commented on his "key insights" and "surprising" results.

Ranganathan has presented his results at the Algebra, Number Theory and Combinatorics Seminar of the Claremont Colleges, the 2011 Joint Mathematics Meetings, and the Western Algebraic Geometry Seminar. Ranganathan is a 2012 graduate of Harvey Mudd College. 


\section{Biographical Sketch}

Dhruv Ranganathan grew up in Chennai, India, and Johannesburg, South Africa. Despite early aspirations to be a professional cricket player, his interest in mathematics and physics quickly took over. It was at the age of nine that he began learning advanced mathematics outside of school, under the private tutelage of A. V. Ramamoorthy in Chennai. At Harvey Mudd College, he worked closely with Dagan Karp for over two years, studying Gromov-Witten theory and toric geometry. This project shaped his current research interests, which include algebraic geometry, toric and tropical geometry, and high-energy physics. Ranganathan is a winner of the Giovanni Borrelli Fellowship and was awarded the Chavin Prize for his Harvey Mudd College senior thesis entitled "Gromov-Witten theory of blowups of toric threefolds". He is currently a first-year graduate student at Yale University. When not studying mathematics, Ranganathan can be found playing the violin, finding innovative ways to cook for himself, or honing his flying trapeze skills.

\section{Response from Dhruv Ranganathan}

I am deeply humbled and honored to be selected for Honorable Mention for the 2013 Morgan Prize. I thank the AMS, MAA, and SIAM for selecting me. I owe a debt of gratitude to my advisor Dagan Karp for his friendship, guidance, and patience. I also express my gratitude to my teachers, Alfonso Castro, Jon Jacobsen, Michael Orrison, and A. V. Ramamoorthy, all of whom have inspired me to study mathematics. Finally, I owe a massive thank you to my family and friends for their ever-present belief, unquestioning support, and unbounded love.

\section{Citation for Honorable Mention: Jonathan Schneider}

Honorable mention goes to Jonathan Schneider for the 2013 AMS-MAA-SIAM Frank and Brennie Morgan Prize for Outstanding Research in Mathematics by an Undergraduate Student. Schneider is recognized for his solution to a problem posed by Dr. Richard Stanley concerning polynomial sequences of binomial type. One letter writer describes Schneider's “ingenious insights and arguments" as having "vast potential for applications and generalizations." Jon is currently a senior at the Massachusetts Institute of Technology.

\section{Biographical Sketch}

Jon Schneider was born in Montreal, Canada, and grew up in Toronto. His high school mathematics teachers at the University of Toronto Schools encouraged Jon's passion for math, and while at high school Jon represented Canada three times at the International Mathematics Olympiad.

Jon then enrolled at MIT, where he is currently concentrating in mathematics and theoretical computer science. During his freshman summer, Jon was introduced to the world of mathematics research through MIT's Summer Program in Undergraduate Research, where he studied the combinatorics of chip-firing and hyperplane arrangements. The next summer, Jon did research with Richard Stanley, solving an open problem regarding the coefficients of chromatic polynomials of certain graphs. Jon presented his solution to this problem at the 2011 Joint Mathematics Meetings, for which he was recognized with an MAA Undergraduate Poster Prize. Next year Jon intends to attend graduate school in either mathematics or computer science.

\section{Response from Jon Schneider}

I would like to thank the AMS, MAA, and SIAM for selecting me for this honorable mention.

I am especially grateful to Professor Richard Stanley for introducing me to this problem and for advising me over the course of my research. I am further thankful to all of my math professors and classmates at MIT, from whom I have learned a great deal. Finally, I would like to thank my parents for their incredible love and support.

\section{About the Prize}

The Morgan Prize is awarded annually for outstanding research in mathematics by an undergraduate student (or students having submitted joint work). Students in Canada, Mexico, or the United States or its possessions are eligible for consideration for the prize. Established in 1995, the prize was endowed by Mrs. Frank (Brennie) Morgan of Allentown, Pennsylvania, and carries the name of her late husband. The prize is given jointly by the AMS, the Mathematical Association of America (MAA), and the Society for Industrial and Applied Mathematics (SIAM) and carries a cash award of US\$1,200.

Recipients of the Morgan Prize are chosen by a joint AMS-MAA-SIAM selection committee. For the 2013 prize, the members of the selection committee were Colin C. Adams, Jill Dietz, Kathleen R. Fowler, Reza Malek Madani, Kannan Soundararajan, and Sergei Tabachnikov.

Previous recipients of the Morgan Prize are Kannan Soundararajan (1995), Manjul Bhargava (1996), Jade Vinson (1997), Daniel Biss (1998), Sean McLaughlin (1999), Jacob Lurie (2000), Ciprian Manolescu (2001), Joshua Greene (2002), Melanie Wood (2003), Reid Barton (2005), Jacob Fox (2006), Daniel Kane (2007), Nathan Kaplan (2008), Aaron Pixton (2009), Scott Duke Kominers (2010), Maria Monks (2011), and John Pardon (2012).

- Elaine Kehoe 